\title{
Lazy Mobile Intruders ${ }^{\star}$
}

\author{
Sebastian Mödersheim, Flemming Nielson, and Hanne Riis Nielson \\ DTU Compute, Denmark
}

\begin{abstract}
We present a new technique for analyzing platforms that execute potentially malicious code, such as web-browsers, mobile phones, or virtualized infrastructures. Rather than analyzing given code, we ask what code an intruder could create to break a security goal of the platform. To avoid searching the infinite space of programs that the intruder could come up with (given some initial knowledge) we adapt the lazy intruder technique from protocol verification: the code is initially just a process variable that is getting instantiated in a demand-driven way during its execution. We also take into account that by communication, the malicious code can learn new information that it can use in subsequent operations, or that we may have several pieces of malicious code that can exchange information if they "meet". To formalize both the platform and the malicious code we use the mobile ambient calculus, since it provides a small, abstract formalism that models the essence of mobile code. We provide a decision procedure for security against arbitrary intruder processes when the honest processes can only perform a bounded number of steps and without path constraints in communication. We show that this problem is NP-complete.
\end{abstract}

\section{Introduction}

Mobile Intruder. With mobile intruder we summarize the problem of executing code from an untrusted source in a trusted environment. The most common example is executing code from untrusted websites in a web browser (e.g., in Javascript). We trust the web browser and surrounding operating system (at least in its initial setup), we have a security policy for executing code (e.g., on access to cookies in web-browsers), and we want to verify that an intruder cannot design any piece of code that would upon execution lead to a violation of our security policy [11. There are many similar examples where code from an untrusted source is executed by an honest host such as mobile phones or virtual infrastructures.

Related Problems. The mobile intruder problem is in a sense the dual of the mobile agents problem where "honest" code is executed by an untrusted environment [3]. The mobile intruder problem has also similarities with the proofcarrying-code (PCC) paradigm [15]. In PCC we also want to convince ourselves

\footnotetext{
* The research presented in this paper has been partially supported by MT-LAB, a VKR Centre of Excellence for the Modelling of Information Technology. The authors thank Luca Viganò and the anonymous reviewers for helpful comments.
} 
that a piece of code that comes from an untrusted source will not violate our policy. In contrast to PCC, we do not consider a concrete given piece of code, but verify that our environment securely executes every piece of code. Also, of course, we do not require code to be equipped with a proof of its security.

The Problem and a Solution. The difficulty in verifying a given architecture for running potentially malicious code lies in the fact that there is an infinite number of programs that the intruder can come up with (given some initial knowledge). Even bounding the size of programs (which is hard to justify in general), the number of choices is vast, so that naively searching this space of programs is infeasible.

Our key observation is that this problem is very similar to a problem in protocol verification and that one may use similar verification methods to address it. The similar problem in protocol verification is that the intruder can at any point send arbitrary messages to honest agents. Also here, we have an infinite choice of messages that the intruder can construct from a given knowledge, leading to an infinitely branching transition relation of the system to analyze. While in many cases we can bound the choice to a finite one without restriction 4, the choice is still prohibitively large for a naive exploration.

In order to deal with this problem of large or infinite search spaces caused by the "prolific" intruder, a popular technique in model checking security protocols is a constraint-based approach that we call the lazy intruder [121316 6]. In a state where the intruder knows the set of messages $K$, he can send to any agent any term $t$ that he can craft from this knowledge, written $K \vdash t$. To avoid this naive enumeration of choices, the lazy intruder instead makes a symbolic transition where we represent the sent message by a variable $x$ and record the constraint $K \vdash x$. During the state exploration, variables may be instantiated and the constraints must then be checked for satisfiability. The search procedure thus determines the sent message $x$ in a demand-driven, lazy way.

A basic idea is now that code can be seen as a special case of a message and that we may use the lazy intruder to lazily generate intruder code for us. There are of course several differences to the problem of intruder-generated message, because code has a dynamic aspect. For instance the code can in a sense "learn" messages when it is communicating with other processes and use the learned messages in subsequent actions. Another aspect is that we want to consider mobility of code, i.e., the code may move to another location and continue execution there. We may thus consider that code is bundled with its local data and move together with it, as it is the case for instance on migration operations in virtual infrastructures. As a result, when two pieces of intruder-generate code are able to communicate with each other, then they can exchange all information they have gathered. An example is that an intruder-generated piece of code is able to enter a location, gather some secret information there, and return to the intruder's home base with this information.

Contribution. The key idea of this paper is to use the lazy intruder for the malicious mobile code problem: in a nutshell, the code initially written by the 
intruder is just a variable $x$ and we explore in a demand driven, lazy way what this code could look like more concretely in order to achieve an attack.

Like in the original lazy intruder technique, we do not limit the choices of the intruder, but verify the security for the infinite set of programs the intruder could conceive. Also, like in the lazy intruder for security protocols, this yields only a semi-decision procedure for insecurity, because there can be an unbounded number of interactions between the intruder and the environment; this is powerful enough to simulate Turing machines. However by bounding the number of steps that honest processes can perform, we obtain a decision procedure. We show that this problem is NP-complete.

For such a result, we need to use a formalism to model the mobile intruder code - or several such pieces of code - and the environment where the code is executed. In this paper we choose the mobile ambient calculus, which is an extension of common process calculi with a notion of mobility of the processes and a concept of boundaries around them, the ambients. The reason for this choice is that we can develop our approach very abstractly and demonstrate how to deal with each fundamental aspect of mobile code without committing to a complex formalization of a concrete environment such as a web-browser running Javascript or the like. In fact, mobile ambients can be regarded as a "minimal" formalism for mobility. Moreover, it has a well-defined semantics which is necessary to formally prove the correctness of our lazy mobile intruder technique. We therefore avoid a lot of technical problems that are immaterial to our ideas, and neither do we tie our approach to one particular application field.

\section{The Ground Model}

\subsection{The Ambient Calculus}

We use the ambient calculus as defined by Cardelli and Gordon [8]. There is a basic version and an extension with communication primitives; we present the ambient calculus right away with communication and only mention that our method also works, mutatis mutandis, for the basic ambient calculus. Fig. 1 contains the syntax of the ambient calculus, and Fig. 2 and 3 give the semantics by defining a structural congruence $\equiv$ and reduction relation $\rightarrow$, respectively. In these figures, we have already omitted some primitives that we do not consider in this paper, namely replication, name restriction, and path constraints; we discuss these restrictions in Sec. 2.5.

The ambient calculus is an extension of standard process calculi with the usual constructs 0 for the inactive process, $P \mid Q$ for the parallel composition of processes $P$ and $Q$, as well as input $(x) . P$ - binding the variable $x$ in $P$ and output $\langle M\rangle$. In addition we have a concept of a process running within a boundary, or ambient, denoted $n[P]$, and this ambient has the name $n$. For instance one may model by $m\left[P\left|v_{1}[R]\right| v_{2}[Q]\right]$ a situation where a process $P$ is running on a physical machine $m$ together with virtual machines $v_{1}$ and $v_{2}$ that host processes $R$ and $Q$, respectively. The communication rule (4) in Fig. 3 


$$
\begin{aligned}
P, Q & :=\quad \text { processes } \\
& 0 \quad \text { inactivity } \\
& P \mid Q \text { composition } \\
& M[P] \text { ambient } \\
& M . P \text { capability action } \\
& (x) . P \text { input action } \\
& \langle M\rangle \text { output action }
\end{aligned}
$$

Fig. 1. Considered fragment of the ambient calculus

$$
\begin{gathered}
P \equiv P \quad \frac{P \equiv Q}{Q \equiv P} \quad \frac{P \equiv Q \quad Q \equiv R}{P \equiv R} \quad \frac{P \equiv Q}{P|R \equiv Q| R} \\
\frac{P \equiv Q}{M[P] \equiv M[Q]} \quad \frac{P \equiv Q}{M . P \equiv M \cdot Q} \quad \frac{P \equiv Q}{(x) \cdot P \equiv(x) \cdot Q} \quad P|Q \equiv Q| P \\
(P \mid Q)|R \equiv P|(Q \mid R) \quad P \mid 0 \equiv P
\end{gathered}
$$

Fig. 2. Structural congruence relation

for instance says that processes can communicate when they run in parallel, but not when they are separated by ambient boundaries. Process can move with the operations in $n$ and out $n$ according to rules (1) and (2); also one process can dissolve the boundary $n[\cdot]$ of another parallel running ambient by the action open $n$ according to rule (3). In all positions where names can be used, we may also use arbitrary capabilities $M$, e.g., one may have strange ambient names like in in $n$, but this is merely because we do not enforce any typing on the communication rules, and we will not consider this in examples.

We require that in all processes where two input actions $(x) . P$ and $(y) . P$ occur, different variable symbols $x \neq y$ are used. This is not a restriction since we do not have the replication operator and can therefore make all variables disjoint initially by $\alpha$-renaming.

\subsection{Transition Relation}

The definition of the reduction relation $\rightarrow$ in Fig. 3 is standard, however there is a subtlety we want to point out that is significant later when we go to a symbolic relation $\Rightarrow$. The point is that, to be completely precise, the symbols $n, m, P, Q, R, P^{\prime}$, and $Q^{\prime}$ in these rules are meta-variables ranging over names and processes, respectively. When applying a rule, these variables are supposed to be matched with the process they are applied to.

To work with the symbolic approach later more easily, let us reformulate this and make explicit the matching by interpreting rules as rewriting rules. In this 


$$
\begin{gathered}
n[\text { in m.P } \mid Q] \mid m[R] \rightarrow m[n[P \mid Q] \mid R] \\
m[n[\text { out } m . P \mid Q] \mid R] \rightarrow n[P \mid Q] \mid m[R] \\
\text { open } n . P|n[Q] \rightarrow P| Q \\
(x) . P \mid\langle M\rangle \rightarrow P\{x \mapsto M\}
\end{gathered}
$$

Fig. 3. Reduction relation of the ambient calculus

view, the rules (1)-(4) of Fig. 3 define the essential behavior of the in, out, and open operators and communication, while the other rules simply tell us to which subterms of a process the rules may be applied. For instance, the process M.P does not admit a reduction, even if the subterm $P$ does. We can capture that by an evaluation context defined as follows:

$$
\begin{aligned}
& C[\cdot]::=\quad \text { context } \\
& C[\cdot] \perp P \quad \text { empty context } \\
& M[C[\cdot]] \quad \text { ambient context }
\end{aligned}
$$

We define that each rule $r=L \rightarrow R$ of the first four rules of Fig. 3 (where the processes $L$ and $R$ have free (meta-) variables on the left-hand and right-hand side) induces a transition relation on closed processes as follows: $S \rightarrow_{r} S^{\prime}$ holds iff there is an evaluation context $C[\cdot]$ and a substitution $\sigma$ for all the variables of $r$ such that $S \equiv C[\sigma(P)]$ and $S^{\prime}:=C[\sigma(R)] 1$

\subsection{Ground Intruder Theory}

We now define how the intruder can construct processes from a given knowledge $K$, which is simply a set of ground capabilities (i.e. without variables). This model is defined in the style of Dolev-Yao models of protocol verification as the least closure of $K$ under the application of some operators. These operators are encryption and the like for protocol verification, and here they are the following constructors of processes and capabilities (written with their arguments for readability):

$$
\Sigma_{p}=\{0, P \mid Q, M[P], M . P,\langle M\rangle \text {, in } M \text {, out } M \text {, open } M\}
$$

We here leave out the input $(x) . P$ because it is treated by a special rule.

Fig. 4 inductively defines the ground intruder deduction relation $K \vdash_{V} T$ where $K$ is a set of ground capabilities, $T$ ranges over capabilities and processes,

\footnotetext{
${ }^{1}$ One may additionally allow here that $S^{\prime}$ can be rewritten modulo $\equiv$ to match the rules of Fig. 3 precisely, but it is not necessary because when applying further transition rules, this is done modulo $\equiv$.
} 


$$
\begin{aligned}
& \frac{K \vdash M}{K \vdash M}\left(\text { Axiom) } \quad \frac{K \vdash P \quad P \equiv Q}{K \vdash Q}\right. \text { (Str.Cong.) } \\
& \frac{K \vdash_{V_{1}} T_{1} \quad \ldots \quad K \vdash_{V_{n}} T_{n}}{K \vdash_{\cup_{i=1}^{n} V_{i}} f\left(T_{1}, \ldots, T_{n}\right)} f \in \Sigma_{p} \text { (Public Operation) } \\
& \frac{K \vdash_{\{x\}} x}{K} \in \mathcal{V} \quad \text { (Use variables) } \quad \frac{K \vdash_{V} P}{K \vdash_{V \backslash\{x\}}(x) . P} \text { (Input) }
\end{aligned}
$$

Fig. 4. Ground intruder deduction rules

and $V$ is a set of variables such that $V=f v(T)$ the free variables of $T$. We require that the knowledge $K$ of the intruder contains at least one name $k_{0}$, so the intruder can always say something. For $V=\emptyset$ we also write simply $K \vdash T$. Let $\mathcal{V}$ denote the set of all variable symbols. The (Axiom) and (Str.Cong.) express that the derivable terms contain all elements of the knowledge $K$ and are closed under structural congruence. The (Public Operation) rule says that derivability is closed under all the operators from $\Sigma_{p}$; here the free variables of the resulting term are the union of the free variables of the subterms. The rule (Use variables) and (Input) together allow the intruder to generate processes that read an input and then use it.

As an example, given intruder knowledge $K=\{$ in $n, m\}$ we can derive for instance $K \vdash m[(x)$.in n.out $x$.〈open $m\rangle]$.

We use the common term "ground intruder" and later "ground transition system" from protocol verification, suggesting we work with terms that contain no variables. However, we allow the intruder to create processes like $(x) . P$ where $P$ may freely contain $x$, and only require that the intruder processes at the end of the day are closed terms (without free variables). We may thus correctly call it "closed intruder" and "closed transition system" but we prefer to stick to the established terms.

\subsection{Security Properties}

We are now interested in security questions of the following form: given an honest process and a position within that process where the intruder can insert some arbitrary code that he can craft from his knowledge, can he break a security goal of the honest process? This is made precise by the following definition:

Definition 1. Let us specify security goals via a predicate attack $(P)$ that holds true for a process $P$ when we consider $P$ to be successfully attacked. We then also call $P$ an attack state. Let $C[\cdot]$ be an (evaluation) context without free variables that represents the honest processes and the position where the intruder can insert code. Let finally $K_{0}$ be a set of ground capabilities. Then the question we want to answer is whether there exist processes $P_{0}$ and $P$ such that $K_{0} \vdash P_{0}$, $C\left[P_{0}\right] \rightarrow^{*} P$ and $\operatorname{attack}(P)$. 
We generalize this form of security questions as expected to the case where the intruder can insert several pieces of code $P_{0}, \ldots, P_{k}$ in different locations, and they are generated from different knowledges $K_{0}, \ldots, K_{k}$, respectively.

There are many ways to define security goals for the ambient calculus, and we have opted here for state-based safety properties rather than observational equivalences. In fact, the most simple goal is that no intruder process may ever learn a secret name $s$. We can thus describe an attack predicate that holds true for states where a secret $s$ has been leaked to the intruder. To do that, let us label all output actions $\langle M\rangle$ that are part of the intruder generated code with superscript $i$ like $\langle M\rangle^{i}$. We formalize that an intruder-generated process has learned the secret $s$ in a state $S$ :

$$
\operatorname{leak}_{\mathrm{s}}(S) \text { iff }\langle s\rangle^{i} \sqsubseteq S .
$$

Here $\sqsubseteq$ denotes the subterm relation.

Another goal is that the intruder code cannot reach a given position of the honest platform. This can be reduced to a secrecy goal - at the destination waits a process that writes out a secret. A more complex goal is containment: a sandbox may host an intruder code and give that code some secret $s$ to compute with, but the intruder code should not be able to get $s$ out of the sandbox. This can again be reduced to secrecy (of another value $s^{\prime}$ ) if outside the sandbox a special ambient $k_{0}\left[\right.$ open $\left.s .\left\langle s^{\prime}\right\rangle\right]$ is waiting. From this ambient an intruder process (who initially knows the name $k_{0}$ ) can obtain secret $s^{\prime}$ if it was able to learn $s$ and get out of the sandbox.

Example 1. As an example let us consider the firewall example from [8]:

$$
\text { Firewall } \equiv w\left[k\left[\text { out w.in } k^{\prime} \text {.in } w\right] \mid \text { open } k^{\prime} \text {.open } k^{\prime \prime} .\langle s\rangle\right]
$$

The goal is that the firewall can only be entered by an ambient that knows the three passwords $k, k^{\prime}$, and $k^{\prime \prime}$ (in fact having capability open $k$ instead of $k$ is sufficient). Here the ambient $k[\cdot]$ acts as a pilot that can move out of the firewall, fetch a client ambient (that needs to authenticate itself) and move it into the firewall. Suppose we run Firewall $\mid P$ for some process $P$ that the intruder generated from knowledge $K$ and define as an attack a state in which leak holds. If $K$ includes open $k, k^{\prime}, k^{\prime \prime}$, then we have an attack, since the intruder can generate the process $P \equiv k^{\prime}\left[\right.$ open $\left.k \cdot k^{\prime \prime}\left[(x) \cdot\langle x\rangle^{i}\right]\right]$ from $K$. An attack is reached as follows:

$$
\begin{aligned}
& \text { Firewall } \mid P \\
& \rightarrow w\left[\text { open } k^{\prime} \text {.open } k^{\prime \prime} .\langle s\rangle\right] \mid k\left[\text { in } k^{\prime} \text {.in } w\right] \mid P \\
& \rightarrow w\left[\text { open } k^{\prime} \text {.open } k^{\prime \prime} .\langle s\rangle\right] \mid k^{\prime}\left[k\left[\text { in w] } \mid \text { open } k . k^{\prime \prime}\left[(x) .\langle x\rangle^{i}\right]\right]\right. \\
& \rightarrow w\left[\text { open } k^{\prime} \text {.open } k^{\prime \prime} .\langle s\rangle\right] \mid k^{\prime}\left[\text { in } w \mid k^{\prime \prime}\left[(x) \cdot\langle x\rangle^{i}\right]\right] \\
& \rightarrow w\left[\text { open } k^{\prime} \text {.open } k^{\prime \prime} .\langle s\rangle \mid k^{\prime}\left[k^{\prime \prime}\left[(x) \cdot\langle x\rangle^{i}\right]\right]\right] \\
& \rightarrow w\left[\langle s\rangle \mid(x) \cdot\langle x\rangle^{i}\right] \\
& \rightarrow w\left[\langle s\rangle^{i}\right]
\end{aligned}
$$

If the knowledge $K$ from which the intruder process is created does not include open $k$ (or $k$ ), $k^{\prime}$ and $k^{\prime \prime}$, then no attack is possible. Also containment of the secret $s$ in the firewall holds. 


\subsection{The Considered Fragment}

For the automation, we have made some restrictions w.r.t. the original ambient calculus. The replication operator $! P \equiv P \mid ! P$ together with the creation of new names allows for simulating arbitrary Turing machines and thus prevents a decision procedure for security. Similar to the lazy intruder in protocol verification, we thus bound the steps that honest processes can perform and do this by simply disallowing the replication operator for honest processes. Without replication, one of the main reasons for the name restriction operator $\nu$ n.P is gone, since we can $\alpha$-rename all restricted names so that they are unique throughout the processes. Note that the name restriction is also useful for goals of observational equivalence, which are essential for privacy goals [12] but which we do not consider in this paper.

Note that we do not bound the size of processes that the intruder creates: the derivation relation $K \vdash P$ allows him to make arbitrary use of all constructors. It may appear as if the intruder were bounded because $K \vdash P$ does not include the replication operator either, but this is not true: an attack always consists of a finite number of steps (as violation of a safety property) and thus every attack that can be achieved by an intruder process with replication can be achieved by one without replication (just by "unrolling" the replication as much as necessary for the particular attack). The difference between unbounded intruder processes and bounded honest processes thus stems from the fact that we ask questions of the form: "can a concrete honest process (of fixed size) be attacked by any dishonest process (of arbitrary size)?"

We do not need to give the intruder the ability to create arbitrary new names. The reason is that we have no inequality checks in the ambient calculus, i.e., no process can check upon receiving a capability $n$ that it is different from all names it knows (e.g. to prevent replays). Thus, whatever attack works when the intruder uses different self-created names works similarly with always using the same intruder name $k_{0}$ that we give the intruder initially.

Finally, the extension of the mobile ambient calculus with communication includes so-called path constraints of the form $M . M^{\prime}$ that can be communicated as messages. Note that this is not ordinary concatenation of messages (which the symbolic techniques we use can easily handle) but sequences of instructions and only after the first has been successfully executed, the next one becomes available, and so the paths cannot be decomposed. Since this includes several problems that would complicate our method, we have excluded them.

\section{Symbolic Ambients}

We now introduce the symbolic, constraint-based approach that is at the core of this paper. To efficiently answer the kind of security questions we formalized in the previous paragraph, we want to avoid search the space of all processes that an intruder can come up with. To that end, we use the basic idea of the symbolic, constraint-based approach of protocol verification, also known as the lazy intruder [12|13[16]6]. 
When an agent in a protocol wants to receive a message of the form $t$ - a term that contains variables - we avoid enumerating the set of all messages that the intruder can generate and that are instances of $t$ (because this set is often very large or infinite). Rather we remember the constraint $K \vdash t$ where $K$ is the set of messages that the intruder knows at the point when he sends the instance of $t$. We then proceed with states that have free variables, namely the variables of $t$ (and of other messages as they sent and received). The allowed values for these variables are governed by the constraints. For a fixed number of agents and sessions, this gives us a symbolic finite-state transition system. An important ingredient of this symbolic approach is checking satisfiability of the $K \vdash t$ constraints. The complexity of the satisfiability problem has been studied for a variety of algebraic theories of the operators involved, e.g. 910; in the easiest theory, the free algebra, the problem is NP-complete 16 . One can check satisfiability of the constraints on-the-fly and prune the search tree when a state has unsatisfiable constraints. Thus during the search messages get successively instantiated with more concrete messages in a demand-driven, lazy way. Hence the name.

Now we carry over this idea to the ambient calculus and apply it to the processes that were written by the intruder, i.e. lazily creating the intrudergenerated processes during the search. Recall that in the previous section we defined security problems as reachability of an attack state from $C\left[P_{0}\right]$ where $C[\cdot]$ is a given honest agent and $K_{0} \vdash P_{0}$ is any intruder process generated from a given initial knowledge $K_{0}$. We could thus simply work with a symbolic state $C[x]$ where $x$ is a variable and we have the constraint $K_{0} \vdash x$.

There are some inconveniences attached to using variables like this for representing processes. First, with every transition the process changes and we therefore need to introduce new variables and relate them to the old ones. Second, the processes can learn new information by communication with others, so the available knowledge changes. For these two reasons we follow a more convenient option and simply represent an intruder generated process by writing $K$ where $K$ is the knowledge from which it was created. $K$ is a set of capabilities and intuitively $K$ represents any process that can be created from $K$. If a process contains two occurrences of $K$ for the same $K$, they may represent different processes. $K$ may contain variables because we will also handle the communication between processes with the lazy intruder technique. We thus extend the syntax of processes $P, Q$ of Fig. 1 by $K$, and we consider symbolic security problems as reachability of a symbolic attack state (defined in Section 3.2) from an initial state $C[K]$ where $C[\cdot]$ is an honest environment that the intruder code is running in.

A symbolic process will also be equipped with constraints which have the following syntax:

$$
\begin{aligned}
\phi, \psi::= & \text { constraints } \\
K \vdash M & \text { intruder deduction constraint } \\
x=M & \text { substitution } \\
\phi \wedge \psi & \text { conjunction }
\end{aligned}
$$


Intuitively, $K \vdash M$ means that capability/message $M$ can been generated by the intruder from knowledge $K$. In fact, will not use in the symbolic constraints $K \vdash P$ for a process $P$, since we have no construct for sending processes and all processes the intruder generates are thus covered by the $K$ notation.

Semantics. We define the semantics for pairs $(S, \phi)$ of symbolic processes and constraints as a (usually infinite) set of closed processes. An interpretation $\mathcal{I}$ is a mapping from all variables to ground capabilities. We extend this to a morphism on capabilities, processes, and sets of processes as expected, where $\mathcal{I}$ substitutes only free occurrences of variables. We define the model relation as follows:

$$
\begin{array}{lll}
\mathcal{I} \models K \vdash M & \text { iff } & \mathcal{I}(K) \vdash \mathcal{I}(M) \\
\mathcal{I} \models x=M & \text { iff } & \mathcal{I}(x)=\mathcal{I}(M) \\
\mathcal{I} \models \phi \wedge \psi & \text { iff } & \mathcal{I} \models \phi \text { and } \mathcal{I} \models \psi
\end{array}
$$

The semantics of $(S, \phi)$ is the set of possible instantiation of all variables and intruder code pieces $K$ with closed processes:

$$
\begin{aligned}
\llbracket P, \phi \rrbracket & =\{Q \mid \mathcal{I} \models \phi \wedge Q \in \operatorname{ext}(\mathcal{I}(P))\} \\
\operatorname{ext}([K) & =\{P \mid K \vdash P\} \\
\operatorname{ext}(x) & =\{x\} \\
\operatorname{ext}(n) & =\{n\} \\
\operatorname{ext}\left(f\left(T_{1}, \ldots, T_{n}\right)\right) & =\left\{f\left(T_{1}^{\prime}, \ldots, T_{n}^{\prime}\right) \mid T_{1}^{\prime} \in \operatorname{ext}\left(T_{1}\right) \wedge \ldots \wedge T_{n}^{\prime} \in \operatorname{ext}\left(T_{n}\right)\right\}
\end{aligned}
$$

Here the $T_{i}$ range over capabilities and processes and $f$ ranges over all constructors of capabilities and processes. Note the case $\operatorname{ext}(x)$ can only occur when processing a subterm of a process where $x$ is bound, so no free variables occur in any $S_{0} \in \llbracket P, \phi \rrbracket$.

Lazy Intruder Constraint Reduction. A decision procedure for satisfiability of $K \vdash M$ constraints can be designed straightforwardly in the style of [136], since we just need to handle the constructors for capabilities, namely in, out, and open, and we have no destructors (or algebraic properties). The only subtlety here is that we have in general several intruder processes that may learn new capabilities independent of each other and may be unable to exchange with each other what they learned-a multi-intruder problem. That means we cannot rely on the well-formedness assumption often used in the lazy intruder for protocol verification. Suppose the knowledge $K$ in a constraint contains a variable $x$, then well-formedness says that there exists an constraint $K_{0} \vdash M_{0}$ with $K_{0} \subseteq K$ and $M_{0}$ contains $x$, i.e., $x$ is part of a term the intruder generated earlier. Without this assumption, constraint satisfiability is more difficult to check in general [5], however the main problem is the analysis of knowledge $K$ in constraints. This is not an issue because we have no analysis rules for the intruder here. For more details, see the proof of Theorem 1 . 


\subsection{Symbolic Transition Rules}

We now define a symbolic transition relation on symbolic processes with constraints of the form $(S, \phi) \Rightarrow\left(S^{\prime}, \phi \wedge \psi\right)$. Note that the constraints are augmented in every step, i.e., all previous constraints $\phi$ remain and new constraints $\psi$ may be added.

We first want to lift the standard transition rules on ground processes of Section 2.2 to the symbolic level. The idea is to replace the rule matching defined above with rule unification. Recall that above we have essentially defined a transition rule $r=L \rightarrow R$ to be applicable to state $S$ if $S=C[\sigma(L)]$ for some substitution $\sigma$ and evaluation context $C[\cdot \cdot]$. For the symbolic level we have that $S$ may contain free variables that need to be substituted as well. Suppose the rule $r$ does not contain any variables that occur in the symbolic state $(S, \phi)$ (which is achieved by $\alpha$-renaming the rule variables). Thus define that $(S, \phi) \Rightarrow_{r}\left(S^{\prime}, \phi \wedge \psi\right)$ holds iff there is an evaluation context $C[\cdot]$ and a term $T$ such that:

$-S \equiv C[T]$

- $\sigma$ is a most general unifier of $T$ and $L$ modulo $\equiv$, i.e., $\sigma(T) \equiv \sigma(L)$ and for no generalization $\tau$ of $\sigma$ it holds that $\tau(T) \equiv \tau(L)$; and

- $S^{\prime}=\sigma(C[\sigma(R)])$ and $\psi=e q(\sigma)$

where $e q(\sigma)$ is the formula $x_{1}=t_{1} \wedge \ldots x_{n}=t_{n}$ if $\sigma=\left[x_{1} \mapsto t_{1}, \ldots, x_{n} \mapsto t_{n}\right]$. Observe $\sigma$ may now replace also variables that occur in $S$ and thus $\sigma$ is applied also to $C[\cdot]$. Moreover for a given $(S, \phi)$ and rule $r$ there can only be finitely many most general unifiers $\sigma$ as discussed in the proof of Theorem 1

Example 2. Using the in rule, we can now make the following symbolic transition: $(x[P] \mid y[$ in $z \cdot Q], \phi) \Rightarrow(z[P \mid y[Q]], \phi \wedge x=z)$

Similarly, also $(x[P] \mid y[$ in $z . K], \phi) \Rightarrow(z[P \mid y[K]], \phi \wedge x=z)$ is possible for an intruder generated piece of code $K$.

So far, however, the rules do not allow us to make an in transition on the following state: $(x[P] \mid y[K], \phi)$ even if the intruder can generate a process of the form in $z . Q$ from knowledge $K$. We will see below how to add appropriate rules for intruder-generated processes, so that for instance in the above state a variant of the in-rule is applicable.

It is immediate that the described symbolic transitions are sound (i.e., all states that are reachable in the symbolic model represent states that are reachable in the standard ground model). There are however not yet complete: in the condition $S \equiv C[T]$ above we restrict the application of rule $r$ to contexts that exist in $S$-without instantiating intruder code like $K$ first. Giving a complete set of rules for intruder processes is the subject of the rest of this subsection.

Intruder-written Code. We now come to the very core of the approach: lazily instantiating a piece of code $K$ that the intruder generated from knowledge $K$ with a more concrete term in a demand-driven way. This is basically what is 
missing after the lifting of the ground rules that we have just described, namely when an "abstract" piece of intruder-written code $K$ prevents the application of a rule that would be applicable when replacing $K$ with some more concrete process $P$ such that $K \vdash P$. Obviously we would like to identify such situations without enumerating all processes $P$ that can be generated from $K$.

In the example $x[P] \mid y[K]$ we discussed above, we have the following possibility: if the intruder code marked $K$ were to have the shape in $x . Q$, we could apply the in rule and get to the state $x[y[K] \mid P]$, assuming $K \vdash$ in $x$. Note the residual code (inside $y[\cdot]$ after the move) is again something generated from knowledge $K$.

There is a systematic way to obtain all rules that are necessary to achieve completeness, namely by answering the following question: given a symbolic process with constraints $(S, \phi)$, any ground process $S_{0} \in \llbracket S, \phi \rrbracket$, and a transition $S_{0} \rightarrow S_{0}^{\prime}$ what rule do we need on the symbolic level to perform an analogous transition? Thus, we want to reach an $\left(S^{\prime}, \phi \wedge \psi\right.$ ) (in zero or more steps) such that $S_{0}^{\prime} \in \llbracket\left(S^{\prime}, \phi \wedge \psi\right) \rrbracket$. Of course, the rule should also be sound (i.e. all $S_{0}^{\prime} \in$ $\llbracket\left(S^{\prime}, \phi \wedge \psi\right) \rrbracket$ are reachable with ground transition rules from some $\left.S_{0} \in \llbracket(S, \phi) \rrbracket\right)$. Soundness is relatively easy to see, because we need to consider rules only in isolation. We now systematically derive rules for each case of $(S, \phi), S_{0}$, and $S_{0}^{\prime}$ that can occur and thereby achieve a sound and complete set of symbolic transition rules.

Recall that by the definition, for a transition from $S_{0}$ to $S_{0}^{\prime}$ with rule $r=L \rightarrow$ $R$, we need to have an evaluation context $C_{0}[\cdot]$ and a substitution $\sigma$ of the rule variables such that $S_{0}=C[\sigma(L)]$ and $S_{1}=C[\sigma(R)]$.

The symbolic transition rules we have defined above already handle the case that the symbolic state $S$ has the form $S=C^{\prime}[T]$ where $\sigma\left(C^{\prime}[\cot ]\right)=C[\cdot]$ and $\sigma(L) \equiv \sigma(T)$ (as shown in the examples previously) where at a corresponding position a similar rule (under renaming) can be applied without instantiating intruder code. This includes the case that a rule variable $P$ of type process is unified with a piece $K$ of intruder code.

Another case that does not require further work is when the rule match in $S_{0}$ is for a subterm of intruder-generated code, i.e. that is subsumed by some $K$ in the symbolic term $S$. Here we use the fact that intruder deduction is closed under evaluation: if $K \vdash P$ and $P \rightarrow P^{\prime}$, then also $K \vdash P^{\prime}$.

Therefore all remaining cases that we need to handle are where one or more proper subterms of the redex $\sigma(L)$ in $S_{0}$ are intruder-written code that are not trivial, i.e. represent a variable in $L$. We make a case distinction

- by the different transition rules for $\rightarrow$, namely (1)-(4),

- and by how $S$ relates to the matching subterm in $S_{0}$.

In-Rule. Let us mark three positions in the in rule which could be intruderwritten code and that are not yet handled: 


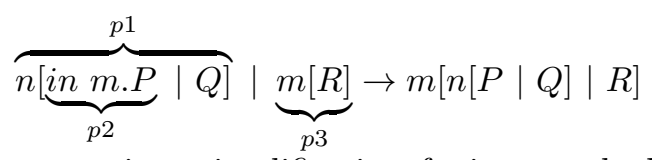

In fact, this notation contains a simplification: for instance looking at position $p_{2}$, we could also have the variant that the intruder code is of the form in m.P $\mid P^{\prime}$. In such a case, the intruder code piece $K$ in the symbolic state would not exactly correspond to a subterm of the matched rule, but only after "splitting" $K$ into $K \mid K$. Such a splitting rule would obviously be sound, but we do not want to include it, and rather perform such splits only in a demand driven way (as the following cases show) - and to keep the notation simple for the positions in the rules. So all positions indicated here are considered under the possibility that the intruder code itself is a parallel composition; note also we are matching/unifying modulo $\equiv$.

In rule with intruder code at position $p_{1}$. The first case we consider is when only at $p_{1}$ is intruder code, i.e., we have some intruder code running in parallel with an ambient $m[R]$; then the intruder code may be able to enter $m$ if it has the capability in $m$. As said before, we could have the case that the intruder code first splits into two parts and only one part enters $m$ while the other part stays outside. This can be helpful if the intruder code does not have the capability out $m$. Since the intruder code can always be trivially 0 if there is nothing to do, it is not a restriction to make the split, so we avoid giving two rules. We obtain:

$$
K|m[R] \Rightarrow K| m[x[K] \mid R] \text { and } \psi=K \vdash \text { in } m \wedge K \vdash x
$$

Here we denote with $\psi$ the new constraints that should be added to the symbolic successor state. $x$ is a new variable symbol (that does not occur so far). The reason for introducing this new symbol $x$ is that a process cannot move without being surrounded by an ambient $n[\cdot]$ construct; as the $n[\cdot]$ of the normal in rule has now become part of the $K$ code, we need to say that the intruder himself created the ambient. As there is no obligation to pick a particular name for that ambient, we simply leave it open and just require the intruder can construct it from knowledge $K$. Note that it would be unsound in general to simplify the right-hand side to $m[K \mid R]$ because the intruder cannot get rid of the surrounding $x[\cdot]$ (even though self-chosen) without another process performing open $x$.

To see the soundness of this rule, consider that the intruder code matched on the left-hand side of the rule should have the form $P_{1} \mid x\left[\right.$ in $\left.m . P_{2}\right]$ for some processes $P_{1}$ and $P_{2}$ generated from knowledge $K$. These are then represented by the two $K$ pieces on the right-hand side of the rule.

In rule with intruder code at position $p_{2}$. Here, intruder code is running inside ambient $n$ that runs in parallel with ambient $m$. The intruder code can move ambient $n$ into $m$, if it has the capability in $m$ :

$$
n[K \mid Q] \mid m[R] \Rightarrow m[n[K \mid Q] \mid R] \text { and } \psi=K \vdash i n m
$$


Note that we could have again the situation that the intruder code is a parallel composition, i.e. of the form in m. $P_{1} \mid P_{2}$. However, then after the move we still have $P_{1} \mid P_{2}$ and we thus do not make the split explicit, because this case is still subsumed by $K$ on the right-hand side.

In rule with intruder code at position $p_{3}$. Now we consider the situation that an honest ambient $n[$ in $m . P \mid Q]$ that wants to enter an ambient $m$ that runs in parallel with intruder code. If the intruder code has name $m$, it can provide the ambient that the honest process can then enter:

$$
n[\text { in } m . P \mid Q]|K \Rightarrow m[n[P \mid Q] \mid K]| K \text { and } \psi=K \vdash m
$$

Here we have again an explicit split of the intruder process into two parts. This is because the concrete intruder process that is partially matched by the left-hand side may have the form $m\left[R_{1}\right] \mid R_{2}$, i.e. not entirely running within $m$, and we thus need to denote that residual process explicitly on the right-hand side.

In rule with intruder code at several positions. If the intruder code is at several positions of the rule, we get the following situations. Obviously we do not need to consider the combination $\left(p_{1}\right)+\left(p_{2}\right)$ because $\left(p_{2}\right)$ is a sub-position of $\left(p_{1}\right)$. The case $\left(p_{1}\right)+\left(p_{3}\right)$ means that we have two intruder processes (in general with different knowledge) to run in parallel: $K \mid K^{\prime}$. We will show below (when we treat communication) that what they can achieve together is to pool their knowledge and join to one process $K \cup K^{\prime}$.

What is left is the combination $\left(p_{2}\right)+\left(p_{3}\right)$ which means that one intruder process runs inside an ambient $n$ and that runs in parallel with another intruder process. This case we can express by the following rule:

$$
n[K \mid Q]\left|K^{\prime} \Rightarrow x\left[n[K \mid Q] \mid K^{\prime}\right]\right| K^{\prime} \text { and } \psi=K \vdash \text { in } x \wedge K^{\prime} \vdash x
$$

Note that the two processes that we start with may not have the same knowledge (here $K$ and $K^{\prime}$ ). Again, we have an explicit split on the side of the $K^{\prime}$-generated process into a part that is entered by $n[\cdot]$ and one that remains outside. Also, again, this rule has a new variable $x$ for the name of the ambient that is entered by $n[\cdot]$; this name needs to be part of $K^{\prime}$ while $K$ only needs to have the in $x$ capability.

This rule is a problem for the termination of our approach. Observe that the left-hand side ambient $n[\cdot]$ occurs identically as a subterm on the right side; so the rule "packs in" the $n[\cdot]$ ambient into another $x[\cdot]$ ambient. We will therefore later show that we can limit the application of this rule without loosing attacks.

Out Rule. For the out rule we have two positions of intruder code to consider:

$$
m[\overbrace{n[\underbrace{\text { out } m . P}_{p 2} \mid Q]}^{p 1} \mid R] \rightarrow n[P \mid Q] \mid m[R]
$$


Out Rule with intruder code at position $p_{1}$. Here we have the situation that the intruder code is within an ambient $m$ and has the capability out $m$. To move parts of the code, the intruder must put it within some ambient $x$ (where $x$ is again a new variable symbol):

$$
m[K] R] \Rightarrow x[K] \mid m[K] R] \text { and } \psi=K \vdash \text { out } m \wedge K \vdash x
$$

Out rule with intruder code at $p_{2}$. This situation is similar except that the intruder code is already contained within an ambient $n$. We then have:

$$
m[n[K \mid Q] \mid R] \Rightarrow n[K \mid Q] \mid m[R] \text { and } \psi=K \vdash \text { out } m
$$

This subsumes also the case that there is intruder code at both in $m$ and in $n$ (i.e. also within what is matched as $R$ here).

Open-Rule. The open rule has also just two positions for intruder code, the opening code and the opened code:

$$
\underbrace{\text { open n.P }}_{p 1}|\underbrace{n[Q]}_{p 2} \rightarrow P| Q
$$

The rules for the intruder code at $p_{1}$ and at $p_{2}$, respectively are immediate:

$$
\begin{aligned}
& K|n[Q] \Rightarrow K| Q \text { and } \psi=K \vdash \text { open } n \\
& \text { open n.P }|K \Rightarrow P| K \text { and } \psi=K \vdash n
\end{aligned}
$$

The case $(p 1)+(p 2)$ is again the case of two parallel communicating processes that is treated next.

Communication Rule. Again there are two possible positions where intruder code could reside, namely as the sender or as the receiver:

$$
\underbrace{(x) \cdot P}_{p 1} \mid \underbrace{\langle M\rangle}_{p 2} \rightarrow P[x \mapsto M]
$$

Communication with the intruder receiving. The intruder can receive a message $M$ from an honest process running in parallel:

$$
K \mid\langle M\rangle \Rightarrow K \cup\{M\}
$$

Here the resulting intruder process has the message $M$ simply added to its knowledge. The idea is that the remaining process can behave like any process that the intruder could have created, if he initially knew $K \cup\{M\}$. To see that this is sound, consider that the intruder process would have the form $(x) . P$ for a new variable $x$ that can occur arbitrarily in $P$. Thus if this process reads $M$, the resulting $P[x \mapsto M]$ is is a process that can be generated from knowledge $K \cup\{M\}$ if $P$ was created from knowledge $K$. 
Communication with the intruder sending. For the case that intruder code sends out a message that is received by an honest process, we can be truly lazy:

$$
(x) . P|K \Rightarrow P| K \text { and } \psi=K \vdash x
$$

Here, we do not instantiate the message $x$ that is being received, we simply add the constraint that $x$ must be something the intruder can generate from knowledge $K$. This is in fact the classic case of the lazy intruder-postponing the choice of a concrete message that the intruder sends to an agent. Since the intruder knowledge contains at least one name, there is always "something to say", but what it is will only be determined if the variable $x$ gets unified later upon applying some rule (which can render the $K \vdash x$ constraint unsatisfiable).

Communication with the intruder both sending and receiving. Finally we have the rule that was mentioned above already: when two intruder processes meet they can exchange their knowledge and work together further on:

$$
K \mid K^{\prime} \Rightarrow K \cup K^{\prime}
$$

This is sound because every $k \in K \backslash K^{\prime}$ can be sent from the first to the second process until we have $K \mid K \cup K^{\prime}$ and then the second part subsumes the first, so we can simplify it to $K \cup K^{\prime}$. Observe that this rule can also be used when we restrict ourselves to the pure ambient calculus without communication: we then simply have two processes in parallel with capabilities $K$ and $K^{\prime}$, respectively, and what they can achieve is anything a process with capabilities $K \cup K^{\prime}$ can achieve (even without communication).

As part of the proof of Theorem 1, we formally show that the set of rules we gave for the symbolic transition system are sound and complete, i.e., they represent exactly the reachable states of the original ground transition system. This proof is found in the extended version of this paper [14, but our systematic development of the rules (i.e., covering each possible case) in this subsection serves as a proof sketch for completeness (and the soundness is straightforward to check for each rule).

\subsection{Security Properties in the Symbolic System}

Before we can state our main result, we need to formally define the properties we can check for in the symbolic system. Right now, we limit ourselves to secrecy goals as a very basic property, and leave the extension to further security properties for future work.

In general for any property that we want to check, we need to be able to express them for both ground and symbolic states, and these definitions for ground and symbolic states must correspond to each other:

Definition 2. We say that a predicate attack $\left(S_{0}\right)$ on closed processes $S_{0}$ and a predicate $\operatorname{ATTACK}(S, \phi)$ on symbolic processes $(S, \phi)$ correspond iff for every $(S, \phi)$ it holds that

$\operatorname{ATTACK}(S, \phi)$ iff exists $S_{0} \in \llbracket S, \phi \rrbracket$ such that $\operatorname{attack}\left(S_{0}\right)$ 
Recall that the attack predicate for secrecy on the ground level was defined as leak $_{\mathbf{s}}\left(S_{0}\right)$ iff $\langle s\rangle^{i} \sqsubseteq S_{0}$. Define the corresponding predicate on the symbolic level:

$\operatorname{LEAK}_{\mathbf{s}}(S, \phi)$ iff exists $K$ such that $K \sqsubseteq S$ and $K \vdash s \wedge \phi$ is satisfiable.

It is immediate that leak $\mathrm{s}_{\mathrm{s}}$ and $\mathrm{LEAK}_{\mathrm{s}}$ correspond: given any $(S, \phi)$ then

$$
\begin{aligned}
\operatorname{LEAK}_{\mathrm{S}}(S, \phi) & \text { iff exist } K, \mathcal{I} . \quad K \sqsubseteq S, \quad \mathcal{I} \models K \vdash s \wedge \phi \\
& \text { iff exist } K, \mathcal{I} . \quad K \sqsubseteq S, \quad \mathcal{I}(K) \vdash s, \mathcal{I} \models \phi \\
& \text { iff exist } \mathcal{I}, C[\cdot] \cdot C\left[\langle s\rangle^{i}\right] \in \operatorname{ext}(\mathcal{I}(S)) \\
& \text { iff exists } S_{0} \cdot S_{0} \in \llbracket S, \phi \rrbracket \text { and } \operatorname{leak}_{\mathbf{s}}\left(S_{0}\right)
\end{aligned}
$$

\subsection{Main Result}

We can now use the symbolic transition system that we have developed using the lazy intruder technique to give a decision procedure for secrecy in our fragment of the ambient calculus without bounding the intruder.

Theorem 1. The following problem is NP-complete. Given

- a name s,

- a closed process $C_{0}[\cdot]$ (in our the supported fragment),

- and a finite set $K$ of ground capabilities as initial intruder knowledge;

exist $P$ and $S_{0}$ such that $K \vdash P, C_{0}[P] \rightarrow^{*} S_{0}$ and leak $_{\mathbf{s}}\left(S_{0}\right)$ ?

The full proof is found in the extended version [14] and we give here only a proof sketch. We can first show that symbolic transition system we have defined is sound and complete, i.e., representing exactly the reachable states of the standard ground transition system. It is immediate that satisfiability of the constraints of our system is in NP (simple guess and check) and that we can polynomially reduce satisfiability of Boolean formulae to satisfiability of constraints. A similar reduction is possible from satisfiability of Boolean formulae to reachability of leak-states in the ground system, showing that this problem is NP-hard. For containment in NP it remains to show that we can restrict the exploration of the (still infinite-state) symbolic transition system such that the length of explored traces is bounded by a polynomial and such that the explored space contains a $\mathrm{LEAK}_{\mathrm{s}}$ state iff one is reachable at all. The idea for this termination proof is that most rules "consume" an action of an honest agent (limiting their applicability); the rules (5) and (9) can be seen as spreading intruder knowledge and this can be bounded because there is an upper bound on what the intruder can learn from honest agents; the application of the rule (8) can be bounded to the number of actions that the honest agents can do (since all other applications are covered by (5) and (9)); and finally the rules (6) and (10) alone cannot be applied indefinitely often without producing a state repetition. 


\subsection{Examples}

Let us reconsider the firewall example from before, and see how a lazy intruder process would find the attack. In contrast to the original specification, we leave open how the intruder process $P$ exactly works, and rather specify that it is some process generated from the initial knowledge $K=\left\{\right.$ in $\left.k, k^{\prime}, k^{\prime \prime}\right\}$ :

$$
\begin{aligned}
& \text { (Firewall } \mid K, \text { true) } \\
& \Rightarrow\left(w\left[\text { open } k^{\prime} \text {.open } k^{\prime \prime} .\langle s\rangle\right] \mid k\left[\text { in } k^{\prime} \text {.in } w\right] \mid K \text {, true }\right) \quad \text { by rule (2) } \\
& \Rightarrow\left(w\left[\text { open } k^{\prime} \text {.open } k^{\prime \prime} .\langle s\rangle\right] \mid k^{\prime}[k[\text { in } w] \mid K] \mid K, \phi_{1}\right) \text { by rule (7) } \\
& \Rightarrow\left(w\left[\text { open } k^{\prime} \text {.open } k^{\prime \prime} .\langle s\rangle\right] \mid k^{\prime}[\text { in } w \mid K] \mid K, \phi_{2}\right) \quad \text { by rule (11) } \\
& \Rightarrow\left(w\left[\text { open } k^{\prime} \text {.open } k^{\prime \prime} \cdot\langle s\rangle \mid k^{\prime}[K]\right] \mid K, \phi_{2}\right) \quad \text { by rule (1) } \\
& \Rightarrow\left(w\left[\text { open } k^{\prime \prime} .\langle s\rangle \mid K\right] \mid K, \phi_{2}\right) \quad \text { by rule (3) } \\
& \Rightarrow\left(w[\langle s\rangle \mid K] \mid K, \phi_{3}\right) \quad \text { by rule (12) } \\
& \Rightarrow\left(w[K \cup\{s\}] \mid K, \phi_{3}\right) \quad \text { by rule (13) }
\end{aligned}
$$

where we have collected the constraints $\phi_{1}=K \vdash k^{\prime}, \phi_{2}=\phi_{1} \wedge K \vdash$ open $w$, and $\phi_{3}=\phi_{2} \wedge K \vdash k^{\prime \prime}$. These constraints are satisfiable. This corresponds to the attack we had described on the ground model, only here we found it lazily during the search, rather than specifying the process up front. Another difference to the original trace is that we have an intruder process $K$ remaining at the outermost level the entire time. This reflects that the intruder process could be a parallel composition of two parts only one of which enters the firewall- the position outside the does not have to be "given up" by the intruder.

Ambient in the Middle. The previous example has basically identified how an honest client (authenticating itself by the knowledge of the keys $k, k^{\prime}$, and $k^{\prime \prime}$ ) is supposed to behave, namely Client $\equiv k^{\prime}\left[\right.$ open $\left.k . k^{\prime \prime}\left[C_{0}\right]\right]$ for some process $C_{0}$. We now consider the case that such an honest client and firewall execute in the presence of an intruder process $K$ :

$$
\begin{aligned}
& \text { (Firewall } \mid \text { Client } \mid K \text {, true) } \\
& \Rightarrow\left(\text { Firewall } \mid k^{\prime}\left[\text { open } k . k^{\prime \prime}\left[C_{0}\right] \mid x[K]\right] \mid K, \phi_{1}\right) \\
& \Rightarrow\left(w\left[\text { open } k^{\prime} \text {.open } k^{\prime \prime} .\langle s\rangle\right] \mid k\left[\text { in } k^{\prime} \text {.in } w\right] \mid k^{\prime}\left[\text { open } k . k^{\prime \prime}\left[C_{0}\right] \mid x[K]\right] \mid K, \phi_{1}\right) \\
& \Rightarrow\left(w\left[\text { open } k^{\prime} \text {.open } k^{\prime \prime} .\langle s\rangle\right] \mid k^{\prime}\left[k[\text { in } w] \mid \text { open } k . k^{\prime \prime}\left[C_{0}\right] \mid x[K]\right] \mid K, \phi_{1}\right) \\
& \Rightarrow\left(w\left[\text { open } k^{\prime} \text {.open } k^{\prime \prime} .\langle s\rangle\right] \mid k^{\prime}\left[\text { in } w\left|k^{\prime \prime}\left[C_{0}\right]\right| x[K]\right] \mid K, \phi_{1}\right) \\
& \Rightarrow\left(w\left[\text { open } k^{\prime} \text {.open } k^{\prime \prime} .\langle s\rangle\left|k^{\prime}\left[k^{\prime \prime}\left[C_{0}\right] \mid x[[K]]\right]\right| K, \phi_{1}\right)\right. \\
& \Rightarrow\left(w\left[\text { open } k^{\prime \prime} \cdot\langle s\rangle\left|k^{\prime \prime}\left[C_{0}\right]\right| x[[K]] \mid K, \phi_{1}\right)\right. \\
& \Rightarrow\left(w\left[\langle s\rangle\left|k^{\prime \prime}\left[C_{0}\right]\right| K\right] \mid K, \phi_{2}\right)
\end{aligned}
$$

where $\phi_{1}=K \vdash$ in $k^{\prime} \wedge K \vdash x$ and $\phi_{2}=K \vdash$ in $k^{\prime} \wedge K \vdash k^{\prime \prime}$. Note that in the one but last step we apply the open action to the intruder ambient $x[K]$ (unifying $x=k_{0}$ ). Thus, the intruder can inject code into the firewall (without being isolated by $x[\cdot]$, and so he can obtain $s$ ) if he knows only in $k^{\prime}$ and $k^{\prime \prime}$. The open $k$ capability is not needed, since this is done by the client after the intruder has infected it. 
A Communication Example. As an example where capabilities are communicated consider the process $n_{1}\left[K_{1} \mid n_{2}\left[\right.\right.$ in $n_{3} \cdot\left\langle\right.$ in $\left.\left.\left.n_{4}\right\rangle\right]\right] \mid n_{5}\left[n_{4}\left[\left[K_{2} \mid\left\langle\right.\right.\right.\right.$ out $\left.\left.\left.n_{5}\right\rangle\right]\right]$ where $K_{1}=\left\{n_{3}\right.$,open $\left.n_{2}\right\}$ and $K_{2}=$ \{open $\left.n_{1}\right\}$. Let the goal be that there is no intruder process who will know both open $n_{1}$ and open $n_{2}$. The lazy mobile ambient technique finds an attack as follows:

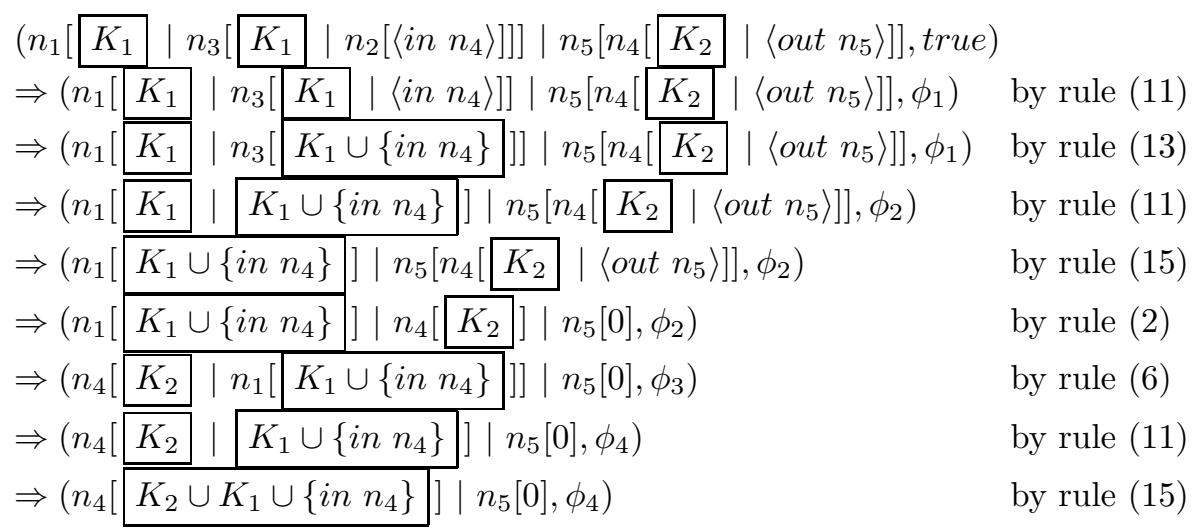

where we the following satisfiable constraints: $\phi_{1}=K_{1} \vdash$ open $n_{2}, \phi_{2}=\phi_{1} \wedge K_{1} \vdash$ open $n_{3}, \phi_{3}=\phi_{2} \wedge K_{1} \cup\left\{\right.$ in $\left.m_{4}\right\} \vdash$ in $m_{4}$, and $\phi_{4}=\phi_{3} \wedge K_{2} \vdash$ open $n_{1}$. We have reached a state where an intruder process knows both open $n_{1}$ and open $n_{2}$.

\section{Conclusions}

We have transferred the symbolic lazy intruder technique from protocol verification to a different problem: an intruder who creates malicious code for execution on some honest platform. This gives us an efficient method to check whether the platform achieves its security goals for any intruder code, because we avoid the naive search of the space of possible programs that the intruder can come up with. Instead we determine this code in a demand-driven, lazy way.

Our approach is closest to a model-checking technique. In contrast to static analysis approaches, it works without over-approximation, but requires a bounding of the number of steps that honest agents can perform. The symbolic nature however allows to work without any bound on the size of programs that the intruder can generate. This is similar to the original use of the lazy intruder in protocol verification 1213166 .

We have used a fragment of the mobile ambient calculus with communication as a small and succinct formalism to model both the platform and the mobile code [8. We have omitted the replication operator in order to bound honest processes (though not the intruder). We have omitted the path constraints because they induce considerable complications for our approach and leave their integration for future work. We also plan to consider the extension of boxed ambients introduced by Bugliesi et al. [7] which add interesting means for access control and communication. Moreover it is possible to extend the ambient calculus and 
our method to support cryptographic operators (like encryption and signing) in the communication of processes.

We believe that the approach we have presented here is generally applicable to the formal analysis of platforms that host mobile code. The key elements can be summarized as follows. First, the code can be lazily developed by exploring at each step which operations can be performed next and what data is needed. This data is handled lazily as well. Second, the intruder code has a notion of knowledge that it can use in further operations and communications, and every received message adds to this knowledge. Third, the code may be able to move to other locations; two pieces of intruder code that meet then pool their knowledge.

\section{References}

1. Abadi, M., Fournet, C.: Mobile values, new names, and secure communication. In: ACM Symposium on Principles of Programming Languages, pp. 104-115 (2001)

2. Abadi, M., Fournet, C.: Private Authentication. Theoretical Computer Science 322(3), 427-476 (2004)

3. Algesheimer, J., Cachin, C., Camenisch, J., Karjoth, G.: Cryptographic security for mobile code. In: IEEE Symposium on Security and Privacy, pp. 2-11 (2001)

4. Arapinis, M., Duflot, M.: Bounding Messages for Free in Security Protocols. In: Arvind, V., Prasad, S. (eds.) FSTTCS 2007. LNCS, vol. 4855, pp. 376-387. Springer, Heidelberg (2007)

5. Avanesov, T., Chevalier, Y., Rusinowitch, M., Turuani, M.: Intruder deducibility constraints with negation. CoRR, abs/1207.4871 (2012)

6. Basin, D., Mödersheim, S., Viganò, L.: OFMC: A symbolic model checker for security protocols. International Journal of Information Security 4(3), 181-208 (2005)

7. Bugliesi, M., Castagna, G., Crafa, S.: Access control for mobile agents: The calculus of boxed ambients. ACM Trans. Program. Lang. Syst. 26(1), 57-124 (2004)

8. Cardelli, L., Gordon, A.D.: Mobile ambients. Theor. Comput. Sci. 240(1), 177-213 (2000)

9. Chevalier, Y., Küsters, R., Rusinowitch, M., Turuani, M.: Deciding the Security of Protocols with Diffie-Hellman Exponentiation and Products in Exponents. In: Pandya, P.K., Radhakrishnan, J. (eds.) FSTTCS 2003. LNCS, vol. 2914, pp. 124135. Springer, Heidelberg (2003)

10. Delaune, S., Lafourcade, P., Lugiez, D., Treinen, R.: Symbolic protocol analysis for monoidal equational theories. Inf. Comput. 206(2-4), 312-351 (2008)

11. Groß, T., Pfitzmann, B., Sadeghi, A.-R.: Browser Model for Security Analysis of Browser-Based Protocols. In: De Capitani di Vimercati, S., Syverson, P.F., Gollmann, D. (eds.) ESORICS 2005. LNCS, vol. 3679, pp. 489-508. Springer, Heidelberg (2005)

12. Huima, A.: Efficient infinite-state analysis of security protocols. In: Proc. FLOC 1999 Workshop on Formal Methods and Security Protocols (1999)

13. Millen, J.K., Shmatikov, V.: Constraint solving for bounded-process cryptographic protocol analysis. In: Proceedings of CCS 2001, pp. 166-175. ACM Press (2001)

14. Mödersheim, S., Nielson, F., Nielson, H.R.: Lazy mobile intruders (extended version). Technical Report IMM-TR-2012-13, DTU Informatics (2012), imm.dtu.dk/ samo

15. Necula, G.C.: Proof-carrying code. In: POPL, pp. 106-119 (1997)

16. Rusinowitch, M., Turuani, M.: Protocol insecurity with a finite number of sessions, composed keys is NP-complete. Theor. Comput. Sci. 1(299), 451-475 (2003) 